\title{
Avaliação rebrota da copa das árvores de pau-rosa (Aniba rosaeodora Ducke) em sistema de podas sucessivas
}

\author{
Paulo de Tarso B. SAMPAIO ${ }^{1}$; Márcia Castro dos SANTOS²; Gil VIEIRA ${ }^{3}$; Wilson SPIRONELLO ${ }^{4}$; Fabio \\ Lozano USECHE5 ; Flávio Mauro Souza BRUNO'

\section{RESUMO} \\ O estudo foi conduzido em um plantio de pau-rosa com 36 anos, localizado na Reserva Florestal Adolpho Ducke, Manaus-AM,

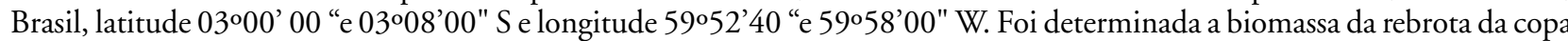 \\ das árvores de pau-rosa (Aniba rosaeodora Ducke, Lauraceae), podadas duas vezes (em 2000 e 2002) e das árvores podadas três vezes \\ (em 1987, 2000 e 20002). Os resultados não indicaram diferenças significativas entre o DAP, altura total, número de brotos/fuste, \\ comprimento e diâmetro dos brotos e peso verde da copa das árvores podadas duas ou três vezes. Os coeficientes de regressão e de \\ correlação de Pearson indicaram que a biomassa da copa das árvores podadas pela terceira vez está fortemente correlacionada com a \\ radiação PAR direta, PAR difusa, $P A R$ total e o índice de área foliar. O elevado número de brotos/fuste revelou boa capacidade da \\ rebrota da copa após sucessivas podas, fato que possibilita o manejo da biomassa das árvores através de podas.
}

PALAVRAS-CHAVE

Biomassa, pau-rosa, luz.

\section{Evaluation of resprouting of rosewood tree (Aniba rosaeodora Ducke) crowns in sucessive prunings}

\section{ABSTRACT}

This study was carried out in a rosewood plantation (36 years-old) located in the Adolpho Ducke Forest Reserve, Manaus, Amazonas,

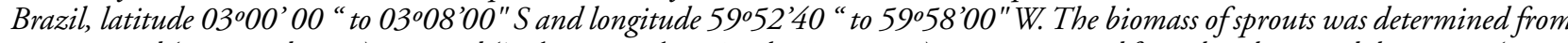
twice pruned (2000 and 2002) rosewood (Aniba rosaeodora Ducke, Lauraceae) tree crowns, and from those harvested three times (1987, 2000 and 2002). The results show that there are no significant differences in biomass productions between trees harvested two or tree times for the following parameters: $D B H$, total height, number of sprouts per bole, sprout length and diameter, and fresh canopy fresh weight. The regression and Pearson correlation coefficients showed that crown biomass from the third pruning was strongly correlated with direct PAR, diffuse PAR and total PAR and LAI (leaf area index). The high number of sprouts per bole reveals the crown's resprouting capacity after successive pruning. This shows that the species can be managed for biomass production through successive pruning.

\section{KEY WORDS}

Biomass, rosewood, light

1Pesquisador INPA/CPST, Manaus, AM, Brasil, sampaio@inpa.gov.br

${ }^{2}$ Enga Florestal, M.Sc.

3Pesquisador INPA/CPST, Manaus, AM, Brasil, gap@inpa.gov.br

${ }^{4}$ Pesquisador INPA/CPST, Manaus, AM, Brasil, spironello@inpa.gov.br

${ }^{5}$ Eng ${ }^{\circ}$ Florestal, M.Sc

${ }^{6}$ Téc. INPA/CPEC 


\section{INTRODUÇÃO}

O manejo de plantios com espécies florestais por meio do corte raso e regeneração por brotação das cepas vem sendo praticado com sucesso para eucaliptos e algumas espécies nativas da Amazônia central (Clay et al., 2000). A condução de povoamentos florestais pela brotação está baseada na capacidade de rebrota das plantas após o corte. A grande vantagem desse método está na capacidade de crescimento inicial e os baixos custos de implantação dos plantios. A alta produtividade inicial é resultante, principalmente, da presença de um sistema radicular já estabelecido, que facilita a absorção de água e nutrientes existentes no solo (Reis \& Reis, 1997).

Estudos indicam a capacidade de rebrota da copa de árvores adultas de pau-rosa (Sampaio, 1987), produtividade de óleo a partir de galhos $(2,2 \%)$, folhas $(1,6 \%)$ e tronco $(1,2 \%)$ (Gottlieb et al., 1964). Logo, o manejo de plantios através da poda da copa das árvores do pau-rosa poderá se constituir como uma alternativa viável para produção de óleo a partir de galhos e folhas. Estudos mais recentes como os de Chaar (2000) e Oashi et al., (1997), além de corroborarem os presentes resultados, ressaltam que o rendimento de óleo sofre significativa variação em função da procedência e época do ano da coleta do óleo.

A quantificação da biomassa de galhos e folhas de pau-rosa será um instrumento útil na avaliação da produtividade de óleo desta espécie. A indústria de óleos aromáticos está novamente em expansão no Amazonas e demandam produtos com qualidade que atendam às exigências do mercado. $\mathrm{O}$ uso de técnicas de manejo que aliem incremento da biomassa aérea (galhos e folhas) aos menores custos, estimulará os produtores a investirem em plantios comerciais. Entretanto, para a implementação de plantios ex situ, é necessário conhecimentos sobre a auto-ecologia da espécie. Mudas de pau-rosa apresentam maior crescimento e sobrevivência em ambientes parcialmente sombreados como os de clareiras naturais e artificiais (Useche, 2003).

Este trabalho teve como objetivo avaliar se a capacidade da rebrota da copa das árvores de pau-rosa diminui com o número de podas realizadas; quantificar a biomassa da rebrota num período de 36 meses após a poda; avaliar se a produção de biomassa da rebrota da copa está associada com a: Densidade de Fluxo de Fótons Fotossintéticos (DFFF: em inglês PPDF), Abertura do dossel (\%), Fator Direto e Indireto de Sítio (FDS, FIS: em inglês DSF, ISF), Fator Total de Sítio (FTS: em inglês TSF) e Índice de área foliar (IAF: em inglês LAI).

\section{MATERIAL E MÉTODOS}

O experimento foi conduzido em um plantio de pau-rosa com 36 anos na Reserva Florestal Ducke, situada no km 26 da

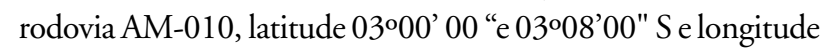
$59^{\circ} 52^{\prime} 40$ "e 59058’00" W. O clima, pela classificação de Koppen é Afi. A temperatura média do mês mais frio nunca é inferior a $18^{\circ} \mathrm{C}$. A precipitação média anual é de $2.186 \mathrm{~mm}$ (Ribeiro $e t$ al., 1984). A vegetação é de floresta tropical úmida de terra firme, com grande diversidade de espécies arbóreas, arbustivas e herbáceas. O solo é do tipo Latossolo Amarelo distrófico de várias texturas (Ranzani, 1980).

Para determinação da biomassa da copa das 20 árvores selecionadas, galhos e folhas de cada árvore foram cortados e pesados separadamente com auxílio de uma balança com capacidade de $300 \mathrm{~kg}$. Para a avaliação da capacidade de rebrota da copa das árvores, foi considerado o número de podas realizadas no mesmo indivíduo em cada tratamento. Em função disso, foram considerados os seguintes fatores: (1) árvores podadas três vezes (1987, 2000 e 2002) e (2) árvores podadas duas vezes (2000 e 2002).

O ambiente lumínico de cada árvore foi determinado através de três fotografias hemisféricas por árvore no mesmo ponto, com auxílio de uma lente Olho de Peixe (fisheye) Nikkor acoplado a uma máquina fotográfica Nikon FM2 com filme FUJI preto e branco ASA 400 (Whitmore et al., 1993). A máquina fotográfica foi acoplada a um tripé nivelado e posicionado a um metro de altura do solo e distante um metro da árvore matriz. O eixo vertical da máquina foi orientado para o Norte magnético com auxílio de uma bússola. As fotografias foram feitas no final da tarde no intervalo das 16:00 às 18:00 horas, com céu encoberto. Os negativos das fotografias foram digitalizados em scanner Sprin Scan 35 Plus, com escala da imagem 256 gray scale na definição de qualidade. Depois de digitalizadas, as fotografias foram preparadas no programa Adobe Photoshop 5.5 para a interpretação dos parâmetros do ambiente lumínico através do programa Hemiphot 1.0 Winphot versão 4.0 (Ter Steege, 1994).

Os parâmetros avaliados para cada tratamento foram: altura total em metros; DAP em centímetros; biomassa total da copa em quilogramas; peso da matéria verde de galhos e folhas em quilogramas; número de brotado; comprimento dos brotos em metros; diâmetro dos brotos em centímetros; Radiação Fotossinteticamente Ativa $(P A R)$ pela medição da Densidade de Fluxo de Fótons Fotossintéticos (PPFD) e parâmetros atribuídos: radiação fotossinteticamente ativa (PAR direta e difusa); Fator Direto de Sítio (DSF) valor atribuído a PAR direta; Fator Indireto de Sítio (ISF) valor atribuído a PAR difusa; Fator Total de Sítio (TSF) valor atribuído a PAR total e Abertura do dossel em percentagem.

O delineamento experimental utilizado foi inteiramente casualizado, com dois tratamentos ( $\mathrm{T}_{1}$ - árvores podadas três vezes $\mathrm{e}_{2}$-árvores podadas duas vezes com 10 repetições por tratamento, totalizando 20 árvores). Para efeito da análise estatística, os dados da radiação fotossinteticamente ativa (PAR direta e difusa), fator direto de sítio (DSF), fator indireto de sítio (DSF), fator total de sítio (TSF), razão vermelho-vermelho extremo (R/FR) e índice de área foliar (LAI) foram submetidos á análise de variância a $5 \%$ de significância. O número de brotos/ 
fuste e DAP também foram avaliados por regressão e correlação da radiação com a biomassa da rebrota das árvores em cada tratamento (Clark, 1961; Tanaka, 1998). O parâmetro percentagem de abertura do dossel foi transformado em arcsen (“' $\mathrm{x} / 100)$.

\section{RESULTADOS E DISCUSSÃO}

Análise de variância não indicou variações significativas entre altura total, DAP, número de brotos/fuste, comprimento e diâmetro dos brotos entre as árvores podadas pela terceira e segunda vez. O desvio padrão da variável número de brotos/ fuste foi elevado $(27,31)$, indicando grande variação na capacidade de rebrota entre os indivíduos estudados (Tabela 1).

Tabela 1 - Comparação de médias pelo teste Tukey para altura total (HT), diâmetro à altura do peito (DAP), número de brotos/fuste, comprimento e diâmetro dos brotos das árvores de pau-rosa (Aniba rosaeodora Ducke) em sistema de plantio sombreado, na Reserva Ducke em Manaus, AM, junho de 2002.

\begin{tabular}{cccccc}
\hline & \multicolumn{2}{c}{ Árvores } & \multicolumn{3}{c}{ Brotações } \\
\hline Tratamento & Altura $(\mathrm{m})$ & DAP $(\mathrm{cm})$ & $\mathrm{N}^{0}$ brotos/fuste & $\begin{array}{c}\text { Comprimento } \\
(\mathrm{m})\end{array}$ & $\begin{array}{c}\text { Diâmetro } \\
(\mathrm{cm})\end{array}$ \\
T1 (3a. Poda) & $19,98^{\mathrm{A}}$ & $16,35^{\mathrm{A}}$ & $26,8^{\mathrm{A}}$ & $1,24^{\mathrm{A}}$ & $1,53^{\mathrm{A}}$ \\
T2 (2a. Poda) & $18,36^{\mathrm{A}}$ & $14,32^{\mathrm{A}}$ & $20,0^{\mathrm{A}}$ & $1,4^{\mathrm{A}}$ & $1,63^{\mathrm{A}}$ \\
F & $0,69^{\mathrm{ns}}$ & $0,58^{\mathrm{ns}}$ & $0,32^{\mathrm{ns}}$ & $0,40^{\text {ns }}$ & $0,10^{\text {ns }}$ \\
S & 5,36 & 5,69 & 27,31 & 0,79 & 6,92 \\
\hline ns = não significativos pelo Teste $\mathrm{F}$ ao nível de $5 \%$ de probabilidade; $\mathrm{s}=$ desvio padrão;
\end{tabular}

Observando as Figuras 1 e 2, existe uma relação positiva entre DAP e o número de brotação/fuste para os dois tratamentos. Nas árvores podadas pela terceira vez, existe uma correlação mediana $(\mathrm{r}=0,69 ; P=0,0385)$ entre o DAP x número de brotos/fuste. Já nas árvores podadas pela segunda vez, essa correlação, é maior $(\mathrm{r}=0,82 ; P=0,0032)$. Estudos anteriores já indicavam que a prática de sucessivas podas da copa das árvores de pau-rosa não comprometia a capacidade de rebrota e que árvores com maior DAP apresentaram maior número de brotaçōes (Sampaio et al., 2000). Árvores podadas pela terceira e segunda vez apresentaram, em média, 26,8 e 20 brotos/fuste respectivamente, indicando que a poda de galhos e folhas estimulou vigorosas brotações das árvores de pau-rosa.

A condução de plantios florestais por meio da poda oferece grandes vantagens em relação à implantação de novos povoamentos, rápido crescimento dos brotos após a poda, eliminação dos custos de preparo da área e plantio, além dos menores níveis de interferência ambiental. O maior crescimento dos brotos em relação às mudas é devido à presença de um sistema radicular já formado das árvores podadas, que facilita a absorção de água e nutrientes existente no solo, além da presença de gemas adventícias e lignotúberos nas bases das árvores (Reis $\&$ Reis, 1997).

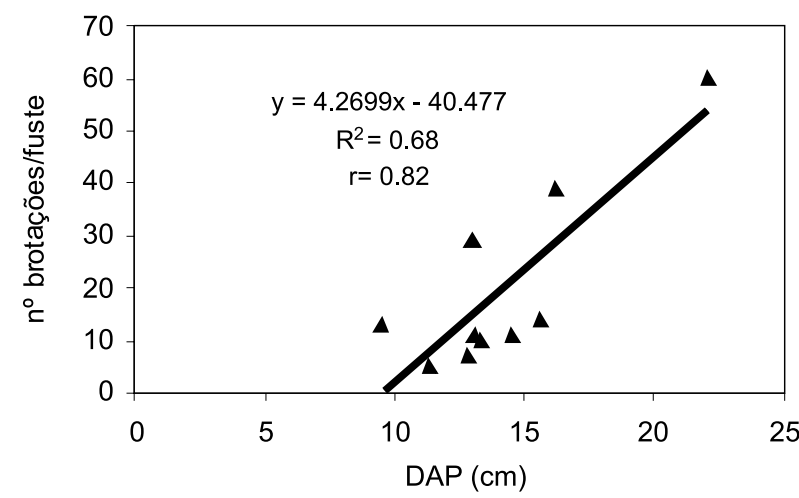

Figura 1 - Relação entre DAP e número de brotações/fuste das árvores da segunda poda. $\mathrm{R}^{2}$ : coeficiente de determinação ajustado $(P=0,0032)$; r: coeficiente de correlação de Pearson $(P=0,0032)$.

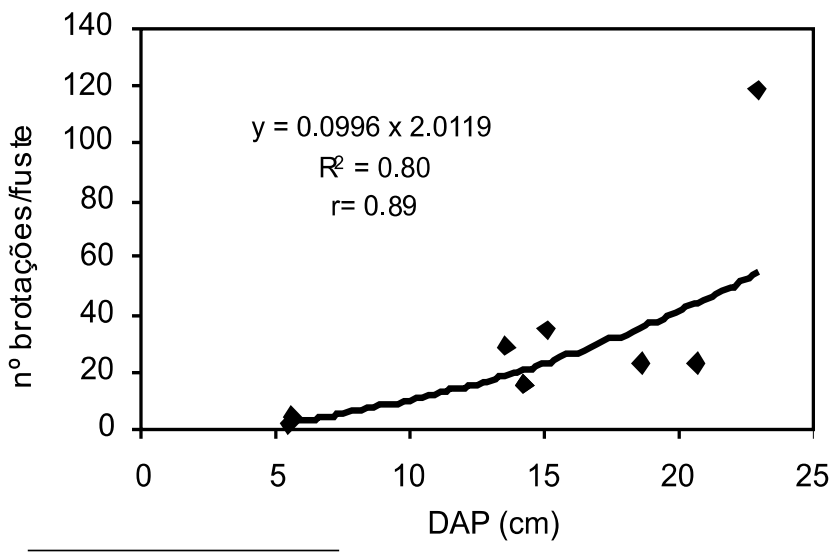

Figura 2 - Relação entre DAP e número de brotações/fuste das árvores da terceira poda. $R^{2}$ : coeficiente de determinação ajustado $(P=00010)$; $r$ : coeficiente de correlação de Pearson $(P=0,0385)$.

Vigorosas brotações após a poda da copa das árvores, interessam ao extrator de óleo, pois a produtividade de óleo é diretamente proporcional á biomassa aérea (Sampaio et al., 2000). Galhos e folhas de pau-rosa apresentam maior produtividade de óleo em relação à madeira desta espécie (Ohashi et al., 1997). Este fato aliado à capacidade de rebrota das árvores podadas, indica a possibilidade do manejo dos plantios através de sucessivas podas.

\section{DETERMINAÇÃO DA BIOMASSA (REBROTA DA COPA)}

O peso verde da rebrota da copa das árvores de pau-rosa podadas pela segunda e terceira vez não diferiram significativamente, indicando que a biomassa de galhos e folhas independe do numero de podas praticadas no mesmo indivíduo (tabela 2).

A média de biomassa de galhos e folhas produzida no intervalo de 13 anos entre a primeira poda em 1987 e a segunda poda em 
2000, foi de 60,18 kg.árvore-1 (Sampaio et al., 2000). Estes mesmos indivíduos, podados pela terceira vez (2002), produziram 15,2 kg.árvore ${ }^{-1}$ após 36 meses (Tabela 2).

Existe grande variação na biomassa de galhos e folhas entre as árvores deste plantio. Assim, 36 meses após a poda, os indivíduos de número $\mathrm{P} 3-55$ e 33 produziram, respectivamente, $53 \mathrm{~kg}$ e $44 \mathrm{~kg}$ de galhos e folhas, enquanto que os indivíduos P2-32 e 53 produziram, aproximadamente, $1 \mathrm{~kg}$ de galhos e folhas no mesmo intervalo de tempo.

Estes resultados indicam a necessidade do manejo destes plantios visando otimizar luz, nutrientes e água possibilitando o desenvolvimento de maior biomassa da copa das árvores. Além destes fatores, deve-se considerar que as diferenças genéticas entre indivíduos reproduzidos sexualmente, podem ter contribuído na variação da biomassa de galhos e folhas entre as árvores.

É certo que o manejo dos plantios de pau-rosa através do sistema da poda da copa das árvores, mostra-se como uma alternativa de uso e conservação desta espécie. A reposição da espécie exigida por lei aliada ao elevado preço do óleo, vem estimulando os produtores rurais para o plantio ex situ. Tais plantios contribuirão para diminuir a pressão da exploração predatória das populaçôes remanescentes existente na Amazônia e diminuirão os custos de produção do óleo essencial.

\section{AVALIAÇÃO DA RADIAÇÃO NA BIOMASSA (REBROTA DA COPA)}

A análise de variância da abertura do dossel, radiação $P A R$ direta, PAR difusa e PAR total e seus respectivos fatores direto, difuso e total (DSF, ISF eTSF), abaixo do dossel, não mostraram diferenças significativas entre tratamentos (Tabela 3 ).

Tabela 2 - Comparação de médias pelo teste Tukey do peso verde da copa das árvores de pau-rosa (Aniba rosaeodora Ducke), em sistema de plantio sombreado, na Reserva Ducke em Manaus, AM, junho de 2002.

\begin{tabular}{cc}
\hline Tratamento & Peso verde de galhos e folhas $(\mathbf{k g})$ \\
\hline T1 (3a. Poda) & $15,2^{\mathrm{A}}$ \\
T2 (2a. Poda) & $13,0^{\mathrm{A}}$ \\
F & $0,10^{\text {ns }}$ \\
\hline
\end{tabular}

ns $=$ não significativo pelo Teste $\mathbf{F}$ ao nível de $5 \%$ de probabilidade; $\mathbf{X}=$ média geral dos tratamentos
Os valores dos parâmetros da Tabela 3, coletados abaixo do dossel do plantio de pau-rosa são inferiores a PAR direta (39,23 mol. $\left.\mathrm{m}^{-2} \cdot \mathrm{dia}^{-1}\right)$, PAR difusa $\left(5,88 \mathrm{~mol} \cdot \mathrm{m}^{-2} \cdot \mathrm{dia}^{-1}\right)$ e PAR total $\left(45,11 \mathrm{~mol} \cdot \mathrm{m}^{-2} \cdot \mathrm{dia}^{-1}\right)$ coletados acima do dossel do plantio. Resultados similares foram obtidos por Useche (2003), que observou o mesmo nível de radiação direta e indireta sob o dossel de clareiras artificiais $\left(88\right.$ a $\left.914 \mathrm{~m}^{2}\right)$ no parque fonológico da Estação Experimental de Silvicultura Tropical do INPA.

A Figura 3 mostra os resultados da relação entre a biomassa da copa com a radiaçấo direta, difusa e total. Predominantemente as árvores que apresentaram maior biomassa da copa estão sob maior radiação PAR direta e total abaixo do dossel. É visível que as árvores dominadas desenvolveram menor biomassa da copa em relação às árvores exposta a maior radiação, evidenciando que a biomassa da copa das árvores de pau-rosa é fortemente influenciada pela radiação direta e total.

Resultados similares foram observados por Useche (2003) na avaliação do estabelecimento e desenvolvimento inicial de plântulas de pau-rosa em clareiras artificiais, encontrou que o Índice de ganho foliar, assim como o crescimento absoluto e relativo foi maior em clareiras de maior tamanho $\left(914 \mathrm{~m}^{2}\right)$ ou, em ambiente com maior intensidade de radiação direta.

Estudos indicam que plantas tropicais desenvolveram maior biomassa aérea quando expostas a maior radiação devido à alta capacidade fotossintética e grande atividade metabólica do

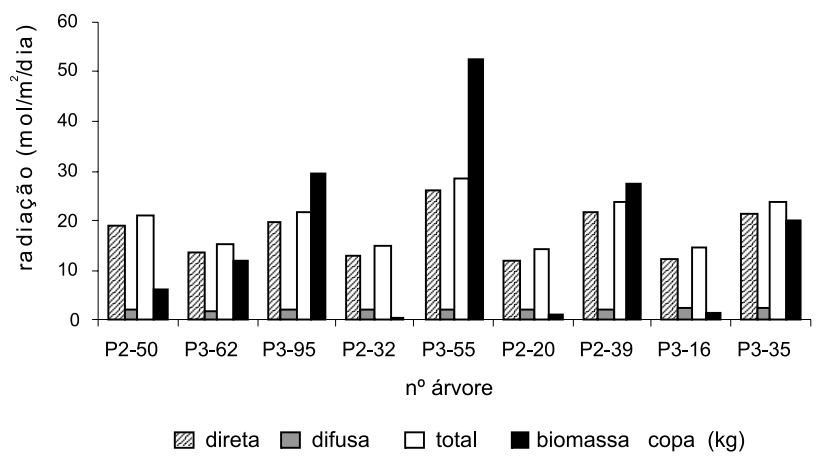

Figura 3 - Radiações diretas, difusas, totais e biomassa da copa para cada árvore de pau-rosa podada pela terceira vez.

Tabela 3 - Análise de variância e comparação de médias pelo teste Tukey para parâmetros da radiação: \%abertura do dossel, PAR direta, PAR difusa, PAR total, fator direto de sítio (DSF), fator indireto de sítio (ISF) e fator total de sítio (TSF).

\begin{tabular}{|c|c|c|c|c|c|c|c|}
\hline Tratamentos & ${ }^{\star}$ PAR direta $(\mathrm{mol} / \mathrm{m} 2 / \mathrm{dia})$ & $\begin{array}{l}\text { *PAR difusa } \\
\text { (mol/m2/dia) }\end{array}$ & $\begin{array}{c}\text { *PAR total } \\
\text { (mol/m2/dia) }\end{array}$ & DSF & ISF & TSF & Abertura do dossel (\%) \\
\hline T1(3a. Poda) & $15,81 \mathrm{~A}$ & $2,07 \mathrm{~A}$ & $17,88 \mathrm{~A}$ & $0,45 \mathrm{~A}$ & $0,35 \mathrm{~A}$ & $0,39 \mathrm{~A}$ & $4,92 \mathrm{~A}$ \\
\hline T2 (2a. Poda) & $17,86 \mathrm{~A}$ & $1,94 \mathrm{~A}$ & $19,80 \mathrm{~A}$ & $0,40 \mathrm{~A}$ & $0,33 \mathrm{~A}$ & $0,44 \mathrm{~A}$ & $5,09 \mathrm{~A}$ \\
\hline $\mathrm{F}$ & $0,48 \mathrm{~ns}$ & $0,25 \mathrm{~ns}$ & $0,37 \mathrm{~ns}$ & $0,52 \mathrm{~ns}$ & $0,22 \mathrm{~ns}$ & $0,39 \mathrm{~ns}$ & $0,09 \mathrm{~ns}$ \\
\hline s & 6,64 & 0,58 & 7,09 & 0,17 & 0,09 & 0,15 & 1,23 \\
\hline$R$ & 2,10 & 0,18 & 2,24 & 0,05 & 0,03 & 0,04 & 0,39 \\
\hline
\end{tabular}

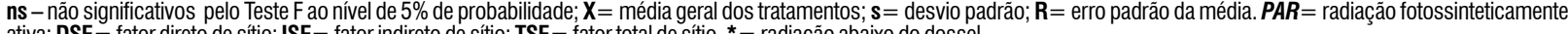
ativa; $\mathbf{D S F}=$ fator direto de sítio; $\mathbf{I S F}=$ fator indireto de sítio; $\mathbf{T S F}=$ fator total de sítio ${ }^{*}=$ radiação abaixo do dossel. 
nitrogênio (Lee, 1988). A maior produção da biomassa de galhos e folhas de plantas submetidas a podas é devido à alta estimulação de gemas dormentes presentes na árvore podada e à necessidade de adaptação ao meio após o processo da poda (Larcher, 2000).

Nas árvores podadas pela segunda vez, a relação entre radiação direta e difusa com a biomassa da copa não foi explícita (Figura 4). Deve-se, portanto, considerar que outros fatores como a umidade do solo e a variabilidade genética entre indivíduos possam exercer forte influência na produção de biomassa aérea dessa espécie.

Análise de regressão - radiação x biomassa (rebrota da copa)

Os resultados das análises de regressão e do coeficiente de correlação de Pearson indicaram forte relação entre os parâmetros da radiação e a biomassa da copa das árvores de pau-rosa podadas pela terceira vez (Tabela 4).

Os coeficientes das regressões e das correlações de Pearson entre biomassa da copa das árvores pela terceira vez e a radiação $P A R$ direta e total demonstram alta correlação (Tabela 4). Espécies de florestas tropicais apresentam maior atividade fotossintética sob maior PAR direta (Ribeiro et al., 1984), fato que explica que árvores de pau-rosa com maior biomassa da copa estão sob maior PAR direta.

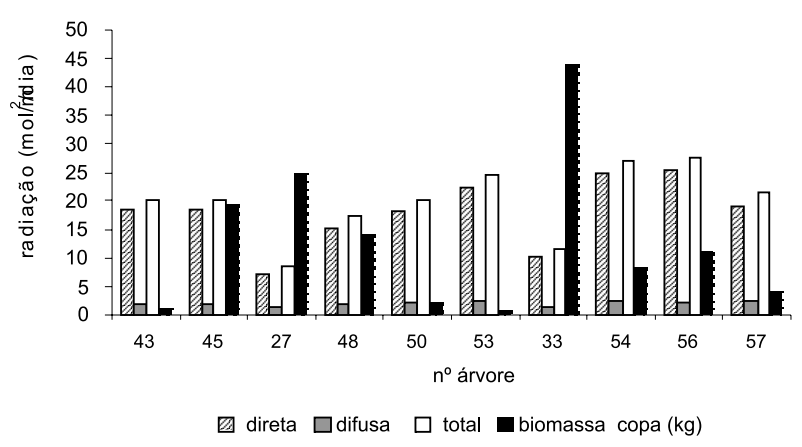

Figura 4 - Radiação diretas, difusas, totais e biomassa da copa para cada árvore de pau-rosa podada pela segunda vez.
Nas árvores podadas pela segunda vez, os coeficientes de regressão indicaram menor relação entre biomassa da copa e fatores da radiação, PAR direta e total (Tabela 5).

Os parâmetros fator direto (DSF), indireto (ISF) e total de sítio (TSF) indicaram forte relação com a biomassa das árvores podadas pela segunda e terceira vez (Tabela 5). O fator direto de sítio (DSF) está relacionado à radiação $P A R$ direta e o fator indireto de sítio (ISF) está relacionado à radiação $P A R$ difusa, sendo que ambos contribuem para o aumento da atividade fotossintética resultando em maior biomassa de galhos e folhas.

\section{CONCLUSÕES}

O elevado número de brotos/fuste das árvores de pau-rosa após a poda, indica que as sucessivas podas praticada no mesmo indivíduo não interferiram na capacidade de rebrota das árvores.

A alta relação entre biomassa da copa e as radiações diretas, difusas e totais, indica que árvores de pau-rosa com maior disponibilidade de luz produziram maior biomassa de galhos e folhas.

A capacidade de rebrota de árvores adultas, aliado à maior produtividade de óleo a partir de galhos e folhas poderão constituir uma alternativa para o manejo, por meio de plantios através de podas sucessivas.

\section{BIBLIOGRAFIA CITADA}

Chaar, J.S. 2000. Estudos analíticos e modificação química por acetilação do linalol contido no óleo essencial da espécie Aniba duckei Kostermans. Tese de Doutorado. Instituto de Química de São Carlos/ Universidade de São Paulo, São Carlos. 125pp.

Clark, F.G. 1961. A hemispherical Forest photocanopymeter. Journal of Forestry, 59(2): 103-105.

Clay, J.W.; P.T.B.; Sampaio, C.R.; Clement, 2000. Biodiversidade Amazônica: exemplos e estratégias de utilização. Instituto Nacional de Pesquisas da Amazônia/SEBRAE, Manaus, Amazonas. 409pp.

Filho, A.O.M.; Wandelli, E.V. 1999. Medidas de radiação solar e índice de área foliar em coberturas vegetais. Acta Amazonica, 29(1): 57-58.

Tabela 5 - Coeficientes de regressão e das correlações de Pearson entre parâmetros da radiação e a biomassa da copa das árvores podadas pela segunda e terceira vez.

\begin{tabular}{|c|c|c|c|c|}
\hline \multirow{2}{*}{ Parâmetros radiação } & \multicolumn{2}{|c|}{ Biomassa da copa (2a. Poda) } & \multicolumn{2}{|c|}{ Biomassa da copa (3a. Poda) } \\
\hline & $\mathrm{R}^{2}$ & $r$ & $\mathrm{R}^{2}$ & $r$ \\
\hline$\%$ abertura do dossel & $0,02(0,6908)$ & $-0,01(0,9769)$ & $0,07(0,4865)$ & $-0,26(0,4865)$ \\
\hline PAR direta & $0,50(0,0217)$ & $-0,68(0,0286)$ & $0,80(0,0010)$ & $0,89(0,0010)$ \\
\hline PAR difusa & $0,69(0,0028)$ & $-0,80(0,0049)$ & $0,008(0,8187)$ & $-0,01(0,9781)$ \\
\hline PAR total & $0,52(0,0181)$ & $-0,69(0,0246)$ & $0,79(0,0012)$ & $0,89(0,7982)$ \\
\hline DSF & $0,50(0,0219)$ & $-0,68(0,0281)$ & $0,81(0,0008)$ & $0,90(0,0008)$ \\
\hline ISF & $0,71(0,0019)$ & $-0,82(0,0035)$ & $0,01(0,7198)$ & $-0,05(0,8814)$ \\
\hline TSF & $0,53(0,0169)$ & $-0,70(0,0235)$ & $0,81(0,0009)$ & $0,90(0,0009)$ \\
\hline
\end{tabular}

$\mathrm{R}^{2}$ : coeficiente de regressão ajustado. r: coeficiente de correlação de Pearson. 0 valor entre parênteses é a probabilidade. PAR: radiação fotossinteticamente ativa; DSF: fator direto de sítio ISF: fator indireto de sítio; TSF: fator total de sítio. 
Gottlieb, O.R.; M. Fineberg, M.L.; Guimarães, M.T.; Magalhães; N. Maravalhas. 1964. Notes on Brazilian Rosewood. Perfumery and Essential Oil Record, 55 (4): 253-257.

Larcher, W. 2000. Ecofisiologia Vegetal. Tradução Carlos Henrique Britto de Assis Prado. São Carlos, RiMa Artes e Textos. 531pp.

Lee, D.W. 1988. Simulating forest shade to study the developmental ecology of tropical plants: juvenile growth in three vines in India. Journal of Tropical Ecology, 4: 281-292.

Ohashi, S.T.; ROSA, L.S.; Santana, J.A. 1997. Brazilian rosewood oil: sustainable production and oil quality management. Perfumer \& Flavorist, 22: 1-5.

Ranzani, G. 1980. Identificação e caracterização de alguns solos da Estação Experimental de Silvicultura Tropical do INPA. Acta Amazonica, 10(1): 7-41.

Reis, G.G.; REIS, M.G.F. 1997. Fisiologia da brotação de eucalyptus com ênfase nas suas relações hídricas. Série Técnica IPEF. $4^{\mathrm{a}}$ Reunião Técnica sobre o Manejo e Brotações de Eucalyptus, 11(30): 121.

Ribeiro, M.N.G.; ADIS. J. 1984. Local rainfall variability - a potential bias for bioecological studies in the Central Amazon. Acta Amazonica, 14 (1-2): 159- 174.

Sampaio, P.T.B.; Vieira, G.; Gomes, L.A.; Leite, A.; Quisen, R. 2000. Regeneração natural como propágulos para produção de mudas de pau-rosa (Aniba rosaeodora Ducke) em viveiro. Resumo VI Congresso e Exposição Internacional Sobre Florestas. Porto Seguro, Bahia, 22 a 26 de outubro, 1:177-180.
Sampaio, P.T.B. 1987. Propagação vegetativa do pau-rosa (Aniba rosaeodora Ducke) pelo método da estaquia. Manaus: INOA. Dissertação de Mestrado, Instituto Nacional de Pesquisas da Amazônia/Universidade Federal do Amazonas. Manaus, Amazonas.

Tanaka, A. 1998. Ecofisiologia do estabelecimento de plantulas em plantios de enriquecimento em Novo Aripuanã. Dissertação de Mestrado, Instituto Nacional de Pesquisas da Amazônia/Universidade Federal do Amazonas. Manaus, Amazonas. 112p.

Ter Steege, H. 1994. Hemiphot (Winphot 4.0), programme to analyze vegetation indices, light in and light quality from hemispherical photographs. Tropenbos Documents, 3.

Useche, F.L. 2003. Estabelecimento e desenvolvimento inicial de plântulas de Aniba rosaeodora Ducke em clareiras artificiais. Dissertação de mestrado, Instituto Nacional de Pesquisas da Amazônia/ Universidade Federal do Amazonas. Manaus, Amazonas. 66pp.

Whitmore, T.C.; Brown, N.D.; Swaine, M.D.; Kennedy, D.; Goodwin-Bailey, C.I.;Gong, W.K. 1993. Use of hemispherical photographs in forest ecology measurement of gap size and radiation total in a Bomean tropical forest. Journal of Tropical Ecology, 9: 131-151.

Recebido em 06/10/2006

Aceito em 08/02/2007 